\title{
Normal Tension Primary Ppen-Angle Glaucoma with Progressive Synechial Angle Closure in Senior Chinese
}

\section{Zhigang Fan ( $\nabla$ fanzhigang@mail.ccmu.edu.cn ) \\ Beijing Tongren Eye Center https://orcid.org/0000-0002-1190-0894}

\section{Zhenni Zhao}

Sun Yat-Sen University Zhongshan Ophthalmic Center

Xiaowei Yu

Sun Yat-Sen University Zhongshan Ophthalmic Center

\section{Dandan Zhang}

Zhengzhou University First Affiliated Hospital

\section{Nannan Sun}

Sun Yat-Sen University Zhongshan Ophthalmic Center

\section{Jiamin Zhang}

Sun Yat-Sen University Zhongshan Ophthalmic Center

\section{Research Article}

Keywords: Normal tension glaucoma, progressive synechial angle closure, combined-mechanism glaucoma, glaucomatous optic neuropathy

Posted Date: May 26th, 2021

DOI: https://doi.org/10.21203/rs.3.rs-538089/v1

License: (c) (7) This work is licensed under a Creative Commons Attribution 4.0 International License. Read Full License 


\section{Abstract}

Background: This study is to address a unique subtype of combined-mechanism glaucoma (CMG), normal tension glaucoma (NTG) with progressive synechial angle closure in the Chinese senior population, whose clinical course and treatment targets are quite different from classic primary angle-closure glaucoma (PACG).

Methods: In this retrospective study, 12 patients with NTG plus primary angle closure suspect or primary angle closure were included in the CMG group, and 12 classic PACG patients with matched extent of glaucomatous optic neuropathy (GON) were included in the control (PACG) group. Clinical features and detailed histories of disease development were investigated and compared between these two groups.

Results: There were 6 females and 6 males in CMG group, 3 males and 9 females in PACG group. No statistical significances were found in age (63.42 $\pm 5.90 \mathrm{vs}$. $59.42 \pm 7.47$ years), preoperative intraocular pressure (20.75 \pm 5.06 vs. $21.25 \pm 4.47 \mathrm{mmHg})$, anterior chamber depth (ACD) (1.98 $\pm 0.28 \mathrm{vs} .1 .94 \pm 0.21 \mathrm{~mm})$, mean deviation of visual field ( $24.37 \pm 6.54$ vs. $22.01 \pm 0.85 \mathrm{~dB}$ ), average retinal nerve fiber layer thickness (48.00 $\pm 14.00 v s .57 .25 \pm$ $14.10 \mu \mathrm{m})$, cup-to-disc (C/D) ratio $(0.89 \pm 0.07$ vs. $0.87 \pm 0.07)$ and axial length $(22.69 \pm 0.76 v s .22 .47 \pm 0.69 \mathrm{~mm})$ between the two groups $(P>0.05)$. The extent of synechial angle closure in PACG group was more extensive than that in CMG group, as evaluated by the number of "clock hours" (3.96 $\pm 1.14 v s .9 .38 \pm 1.99, P<0.05)$, although the severity of GON was comparable.

Conclusion: If NTG develops with synechial angle closure, it might be misdiagnosed as PACG. We need to recognize and scrutinize this specific subtype of $C M G$, which is very common in the Chinese senior population, but widely neglected. In contrast to classic PACG, treatment targets in NTG with progressive synechial angle closure is primarily in consistent with those in NTG.

\section{Introduction}

Primary glaucoma in adults can be classified into open-angle glaucoma and angle-closure glaucoma based on morphology of iridocorneal angle. Essentially, primary open-angle glaucoma (POAG) is defined as "a chronic and progressive optic neuropathy characterized by an acquired atrophy of the optic nerve and a loss of retinal ganglion cells (RGC)", as described in the latest Preferred Practice Pattern (PPP 2016) [1]. Primary angle-closure glaucoma (PACG) is characterized by elevated intraocular pressure (IOP) as a result of mechanical obstruction of the trabecular meshwork by either apposition of the peripheral iris to the trabecular meshwork or synechial angle closure. Importantly, mechanical angle closure is the pathophysiological basis in PACG, so that the range of the angle closure determines the extent of IOP elevation. Intraocular pressure rising to a certain extent and maintaining for a certain period of time would be the direct cause leading to glaucomatous optic neuropathy (GON). PACG can only be diagnosed when GON progresses due to mechanical angle closure. The nomenclature of primary angle closure suspect (PACS) and primary angle closure (PAC) is proposed when evidence of characteristic glaucomatous structural and/or functional damage is not present [2]. The International Society of Geographic and Epidemiologic Ophthalmology (ISGEO) defines PACS as appositional contact between the peripheral iris and posterior trabecular meshwork, PAC as synechial angle closure, and PACG as PAC with evidence of GON [3].

In our daily clinical practice, narrow angle with various degrees of synechial angle closure and GON is quite frequently detected in Chinese elderly Han patients. In many cases, the extent of synechial angle closure is quite limited to only a few clock hours, and IOP is not elevated. By definition from ISGEO and PPP2016, these cases belong to PACG. However, in many ways these cases are quite different from classic PACG. In classic PACG [4], the 
clinical development follows from PACS to PAC, and PACG. Generally, only when synechial angle closure progresses to more than $50 \%$ of the entire angle [5,6], compromised aqueous outflow system leads to gradual IOP elevation which is in proportion to the extent of synechial angle closure and finally cause GON. In our scenario, the degree of GON in many patients is not proportion to the extent of synechial angle closure and IOP elevation, which suggested other underlying pathogenesis in GON progression. The possible explanation for these cases might be that GON happens prior to extensive synechial angle closure. POAG might be the primary diagnosis, and the narrow anterior segment structure leading to progressive synechial angle closure is a coexisting pathology. We define this glaucoma as a unique type of combined-mechanism glaucoma (CMG) $[7,8]$. If this CMG develops with high pressure POAG, the situation might not cause enough alert to differentiate from classic PACG. However, in an extreme condition when this $C M G$ develops in a normal IOP, we will be quite definitive that the condition is primarily normal tension glaucoma (NTG). We believe from our extensive clinical cases, this narrow angle NTG with progressive angle closure is quite common among senior Chinese population. However, the lack of recognition and systemic medical data analysis leads to underdiagnosis.

In our current study, we have retrospectively inspected many glaucoma cases, from which we found 12 cases with solid evidence supporting the existence of NTG with progressive synechial angle closure. We would emphasize the importance of a complete medical database, especially records of series of gonioscopy, UBM and evaluations of GON, as well as IOP in every clinical patient with narrow angle.

\section{Methods}

\section{Ethical approval}

In this retrospective study, patients with complete out-patient medical records tractable to early stage of diseases were selected between November 2015 and November 2020 in Zhongshan Ophthalmic Center, Sun Yat-sen University, China. Institutional approval for this study was obtained from the Ethics committee of the Zhongshan Ophthalmic Center, Sun Yat-sen University, China (No. 2020KYPJ103) and all procedures were conformed to the tenets of the Declaration of Helsinki.

\section{Study population}

To better understand the development of NTG with progressive synechial angle closure and distinguish it with classic PACG, two groups were included in this study. Specifically, NTG with progressive synechial angle closure was included in CMG group, and classic PACG with matched severity of GON was included in PACG group. The diagnosis of PACG, POAG and CMG conformed guidelines from PPP 2016.

Patients who met the following criteria were included into the CMG group. (1) Confirmed POAG diagnosis with functional and/or structural evidence of GON, together with various extent of synechial angle closure; (2) Complete medical history with normal IOP (measured by Goldmann applanation tonometry) records at the early stage of disease prior to the administration of anti-glaucoma treatments. Complete clinical records, including OCT, anterior chamber depth (ACD), visual field (VF), axial length (AL) and optic nerve imaging with vertical cup-to-disc (C/D) ratios were available. Specifically, detailed gonioscopy examination was performed on each patient's eyes using standard procedures. Briefly, the angle recess was evaluated using a 2-mm high, narrow slit beam in the least possible illumination, with the eye in primary gaze. Manipulative and indentation gonioscopy were carried out to visualize all possible angle structures. The presence and extent of peripheral anterior synechiae, trabecular pigmentation, and any other angle abnormalities were recorded. Angles are graded based on Spaeth's classification, 
repeated and confirmed by a senior glaucoma specialist, Dr. Zhigang Fan (FZG); (3) the severity of GON was not consistent with the extent of synechial angle closure (less than 180 degrees) and IOP elevation.

The inclusion criteria of PACG group were as followed. (1) Classic diagnosis of chronic PACG; (2) Complete medical records, showing a progressive synechial angle closure, developing from PACS, PAC to PACG; (3) Severity of GON was in proportion to the extent of synechial angle closure and IOP elevation with extended period of time.

Exclusion criteria in both two group was included as followed. (1) angle synechiae was secondary to other conditions, such as inflammation, neovascularization, and iridocorneal endothelial syndrome (ICE); (2) Systemic or other ocular conditions that affect aqueous outflow function, including Posner-Schlossman syndrome (PSS), trauma or tumor etc.; (3) developmental anomaly of the anterior ocular segments.

A complete medical record for each patient was examined and confirmed by one glaucoma specialist (FZG), including ophthalmic examinations, demographic data, systemic health condition and eye disease history.

\section{Statistical analysis}

Statistical analyses were performed using SPSS software version 22.0 (SPSS, Inc., Chicago, IL, USA). All of the continuous data were recorded as mean \pm standard deviation (SD). The comparisons of age, average RNFL thickness, C/D ratios, AL and MD of VF between two groups were performed using Student $t$ test after the confirmation of homogeneity of variance with an $\mathrm{F}$ test. For all test, $p$-values $<0.05$ was considered to be statistically significant.

\section{Results}

\section{Recruitment and Demographics}

A total of 12 consecutive patients meeting the criteria were selected in the CMG group and 12 consecutive patients diagnosed with PACG during the same period were used as controls. There were 6 males and 6 females in the CMG group, 3 males and 9 females in the PACG group. The age of patients in CMG group and PACG group was $63.42 \pm$ 5.90 and $59.42 \pm 7.47$ years $(P=0.1586)$, respectively. Details are shown in table 1 .

\section{Disease information}

For patients in the CMG group, all 12 patients (100\%) belonged to the advanced stage of glaucoma. For patients in the PACG group, 1 patient (8.33\%) was in the early stage of glaucoma, and 11 patients (91.67\%) were in the advanced stage of the disease. No statistical significances were found between the two groups including IOP before surgery $(20.75 \pm 5.06$ vs. $21.25 \pm 4.47 \mathrm{mmHg}), \mathrm{ACD}(1.98 \pm 0.28$ vs. $1.94 \pm 0.21 \mathrm{~mm}), \mathrm{C} / \mathrm{D}$ ratio $(0.89 \pm 0.07$ vs. 0.87 $\pm 0.07)$ and $\operatorname{AL}(22.69 \pm 0.76$ vs. $22.47 \pm 0.69 \mathrm{~mm})(P>0.05)$ (table 1$)$. Although the severity of GON in two groups was comparable through measuring VF index and RNFL thickness (MD of VF: $24.37 \pm 6.54$ vs. $22.01 \pm 0.85 \mathrm{~dB}, P>$ 0.05 (figure 1A); average RNFL thickness: $48.00 \pm 14.00$ vs. $57.25 \pm 14.10 \mu \mathrm{m}, P>0.05$ ) (figure 1B), the extent of synechial angle closure in PACG group was more extended than that in CMG group showed through the number of "clock hours" (3.96 \pm 1.14 vs. $9.38 \pm 1.99, P<0.05)$ (figure $1 \mathrm{C})$. It is a sign that angle closure contributes only part of the GON defect in CMG patients. When synechial angle closure develops to the extent of more than $50 \%-75 \%$ of the entire angle in PACG patients, aqueous outflow function is compromised, which leads to IOP elevation. Thus, in PACG patients, the extent of synechial angle closure and IOP elevation are consistent, which is also in consistency to the severity of GON defect. Besides, according to patients' history, there was at least one record of characteristic 
structural or functional damage of GON identified in CMG group with normal IOP. While in those classic PACG patients, historical record of normal optic nerve could be tracked from their early disease stage.

\section{Surgical types and prognosis}

In the CMG group, there was 1 patient (8.33\%) accepting the phacoemulsification (phaco) surgery and 2 patients (16.67\%) for trabeculectomy plus phaco and 9 patients (75\%) for trabeculectomy. While in the PACG group, trabeculectomy was performed in 11 patients (91.67\%) and trabeculectomy plus phaco was performed in 1 combined surgery $(8.33 \%)$ which was shown in figure 2 . The IOP of the all patients was decreased significantly after surgery in both two groups (figure 3).

\section{Discussion}

In our current study, PACG group with classic PACG patients and CMG group with patients who had NTG with progressive synechial angle closure were included. Their clinical features and diagnosis were definitive based on a thorough investigation into their disease development history. In the CMG group, the degree of GON damage was severe, but not in proportion to the extent synechial angle closure, which was vital to distinguish from classic PACG.

The most common type of glaucoma in Asian is POAG [9]. NTG constitutes the majority of POAG in Asian population-based studies [10-12], which is one of the distinctive characteristics of glaucoma in Asians compared with other races. The calculated average proportion of NTG in Asia accounted for $76.3 \% \pm 12.4 \%$ of POAG [11], and even up to $80 \%$ - $90 \%$ in Japan and Korea [12], whereas ranged from $30.0 \%$ to $57.1 \%$ in white and African population studies $[13,14]$. In the latest epidemiological survey in the Chinese population, the overall pooled prevalence of NTG among POAG patients was $69.8 \%$ [10]. These studies all revealed that NTG is the most common subtype among patients with POAG in the Chinese population. At the same time, it should be noted that the anatomical features including relatively smaller anterior segments and narrow angle are also common in Asians [15]. Based on the latest epidemiological investigation, approximately $20 \%$ Chinese people aged 50 years and older develop a certain form of angle closure over a 10-year period [16]. Therefore, the prevalence of NTG with PACS/PAC in Chinese elderly people may far exceed our expectations. In our clinical settings, approximately 50\% POAG patients over 60 years old present with some extent of narrow angle, and quite a lot of these senior patients have normal or close to normal IOP. If we do not deliberately scrutinize this special condition from dynamic course of disease development, a misdiagnosis of PACG could be made with pictures in a single disease section.

In Sihota's study, CMG patients with elevated IOP and angle closure less than 90 degrees with a sectional picture of the disease stage were selected. They confirmed that both high-IOP POAG and PACG elements are present in each case [8]. However, there is no history to distinguish which element happens first or both happen simultaneously. In our research, we have specifically investigated medical records of patients who came to our glaucoma clinic over the past 24 months, and identified 12 patients with records that confirmed NTG as initial diagnosis. Follow-up exams testified the development of progressive synechial angle closure. If only through sectional data presented at a later disease stage when an extensive synechial angle closure was already present, our cases would have been indistinguishable to a classic PACG. More importantly, NTG was the primary diagnosis in our particular CMG patients, which determined their target IOP and treatment strategy was quite different from PACG or CMG in Sihota's study. In most of the clinical situations, however, there are no complete records to make a definitive differentiation. Most importantly, no adequate clinical recognition was present among ophthalmologists that this subtype of NTG with progressive synechial angle closure was widely existed in Chinese elderly people. 
In this current study we want to emphasize, firstly that ophthalmologists should get familiar with this specific subtype of CMG, NTG with progressive synechial angle closure. Whenever a diagnosis of PACG is to be made, a scrutinization should be performed examining the consistency on extent of IOP elevation, angle closure and GON. Considering the significantly higher incidence of NTG and narrow angle in Asian population, NTG with narrow angle and progressive synechial angle closure is probably a very common form of glaucoma in Chinese patients. Estimate of its exact incidence is not possible at this moment, due to vastly incomplete medical record due to the current lack of historical medical data system that incorporate medical history with all the imaging examinations. Out of a patient's base of around 1000 CMG during the period of 11/2015-11/2020, we have found 12 cases with tractable records confirming NTG with progressive synechial angle closure. Conclusion with complete scientific evidence demands a longitudinal multi-center clinical trial.

The progressive synechial angle closure is secondary to the original narrow but open angle structure. When there is prolonged and repeated contact between peripheral iris and corneoscleral trabecular meshwork, synechial angle closure develops (<6 hours clocks). For example, our study found a typical patient who had a range of synechial angle closure extending to 2,3 , or 4 clock-hours in the presence of an IOP in the $40 \mathrm{~s} \mathrm{mmHg}$ range. If tonography is taken, it may indicate an impairment of facility of outflow consistent with the IOP, but disproportionate to the extent of angle closure [3]. More importantly, GON damage is much earlier and more severe in consideration of the history of IOP elevation. In classic PACG patients, the extent of synechial angle closure is proportional to the extent and period of IOP elevation. The severity of GON is also consistent with the duration of IOP elevation together with the extent of synechial angle closure. A discrepancy between these three index alerts clinicians to consider the possibility of high-IOP POAG complicated with PACS/PAC. The most definitive evidence differentiating these two categories of patients is a complete investigation of disease history and a clinical picture (medical records) at the early stages of disease is very crucial. In an oversimplified classification of CMG, if GON appears prior to synechial angle closure and elevated IOP, it is NTG with progressive PACS/PAC; if GON is not present in the beginning and develops with the progressive synechial angle closure and elevated IOP, it is classic PACS/PAC/PACG. Therefore, our studies repeatedly address the importance in reporting and investigating the entire disease course. Most importantly, when synechial angle closure and GON are present, every clinician should be prompted to think there are two possible underlying disease pathologies.

The exist of another subtype of CMG, "PACG with secondary trabecular meshwork damage" is an ongoing debate. According to our clinical experience, this situation may exist, but its definitive diagnosis also depends on a complete clinical history and medical records. In this situation, the extent of synechial angle closure is not consistent with the degree of elevated IOP. Hypothetically, appositional contact between peripheral iris and corneoscleral trabecular meshwork causes reduced or complete blockage of aqueous outflow through the specific area of angle, causing functional trabecular meshwork damage. In some patients with elevated IOP, the extent of synechial angle closure is less than 180 degrees, which generally does not cause compromise in aqueous outflow in a typical PAC patient. This also could be misdiagnosed as POAG with PAC/PACS, but if we investigate into the medical history, no initial GON would be identified, which helps to make correct diagnose. A complete clinical record may indicate a progressive synechial angle closure and gradual elevation of IOP, while closed angle complexes the situation. In some clinical cases, there may be a history of repeated episodes of sub-clinical acute or sub-acute angle closure attacks, which may resolve naturally, and may not be serious enough to alert patients seeking immediate medical attention. The hypothesis is that repeated episodes of angle closure attacks cause permanent damage to the corneoscleral trabecular meshwork even without formation of peripheral anterior synechiae (PAS) and that they could in this way induce a form of permanent open-angle glaucoma [3]. This PACS/PAC complicated with functional trabecular meshwork damage, in the perspective of essential disease pathology, should be included into the 
category of PACS/PAC/POAG as a special subtype. Again, complete medical records and patients' history are critical in making the correct diagnosis.

Treatment strategies are quite different between NTG with progressive synechial angle closure and classic PACG. For patients with classic PACG, therapeutic protocols based on the stages of disease [17]. Laser iridotomy or incisional iridectomy is indicated for eyes with PACS and PAC when the extent of synechial angle closure is less than 180 degrees and IOP is not elevated. In some cases, reopening the angle is performed through laser iridoplasty with thickened peripheral iris or surgical separation of synechiae (goniosynechialysis) to improve aqueous outflow, especially when it is performed within 6 to 12 months of an acute angle-closure crisis (AACC) [18-20]. In addition, growing evidence indicates that cataract extraction alone may lead to substantial IOP lowering in some PACG patients and can be considered as an option for treatment. In NTG with progressive synechial angle closure, the treatment should be in consistent with current protocol for NTG as well as the prevention of progressive angle closure. The mainstream treatment for NTG is IOP reduction [20] and aggressive lowering of IOP by $30 \%$ below the baseline can be beneficial [21]. Long-term topical ocular hypotensive agents, incisional surgery, trabeculectomy or tube shunt, and, rarely, laser trabeculoplasty could be selected based on the severity of GON and target IOPs, while the coexisting narrow angle and progressive synechial angle closure should also be addressed simultaneously. Peripheral angle could be opened in some cases with laser iridotomy [22-34]. Following common POAG treatment protocol [35] and our clinical experience with Chinese patients population, IOP should be controlled at $12-18 \mathrm{mmHg}$ in early-median stage, while IOP should be targeted more aggressively at 10-12 mmHg, and even below $10 \mathrm{mmHg}$ in very advanced stage patients. Simultaneously, these patients also need treatment for accompanying PACS/PAC that follows exactly the same strategy and protocol as in a classic PACS/PAC/PACG situation, including iridotomy, iridoplasty or cataract extraction.

In conclusion, we have identified solid evidence, for the first time, for a specific subtype of NTG with progressive synechial angle closure. Scrutinizing medical records to build an entire clinical picture is important for making correct diagnosis. Appropriate therapeutic strategies primarily focus on NTG should be administered supplemented by treatments on PAC/PACS. We look forward to conducting longitudinal multicenter clinical trials to improve our understandings.

\section{Declarations}

Funding: This work was supported by Guangdong Natural Science Foundation (Grant number: 2020A151501168; Guangzhou, China) and Guangzhou Science and Technology Plan Project (Grant number: 202102021099; Guangzhou, China).

Conflicts of interest: The authors declare that they have no conflict of interest.

Compliance with ethical standards: This study was approved by the Ethics Committee of Zhongshan Ophthalmic Center. Besides, this study was performed in accordance with the ethical standards as laid down in the 1964 Declaration of Helsinki and its later amendments or comparable ethical standards.

Availability of data, material and code: All of the data, material and code in this article is availability.

Availability of data, material and code: All of the data, material and code in this article is availability.

Authors' contributions: All authors contributed to the study conception and design. Material preparation, data collection and analysis were performed by Zhenni Zhao and Dandan Zhang. All surgeries were performed by Dr. 
Zhigang Fan. The first draft of the manuscript was written by Zhenni Zhao, Xiaowei Yu and Dandan Zhang and all

authors commented on previous versions of the manuscript. All authors read and approved the final manuscript.

Consent to participate: Informed consent was obtained from all individual participants included in the study.

Consent to publish: Patients signed informed consent regarding publishing their data in this article.

\section{References}

1. Prum BE Jr, Lim MC, Mansberger SL, Stein JD, Moroi SE, Gedde SJ, Herndon LW Jr, Rosenberg LF, Williams RD (2016) Primary Open-Angle Glaucoma Suspect Preferred Practice Pattern((R)) Guidelines. Ophthalmology 123(1):P112-P151. doi:10.1016/j.ophtha.2015.10.055

2. Prum BE Jr, Herndon LW Jr, Moroi SE, Mansberger SL, Stein JD, Lim MC, Rosenberg LF, Gedde SJ, Williams RD (2016) Primary Angle Closure Preferred Practice Pattern((R)) Guidelines. Ophthalmology 123(1):P1-Pp40. doi:10.1016/j.ophtha.2015.10.049

3. Malik Y. Kahook JSS (2013) Chandler and Grant's Glaucoma, Fifth Edition. SLACK Incorporated

4. Foster PJ, Buhrmann R, Quigley HA, Johnson GJ (2002) The definition and classification of glaucoma in prevalence surveys. Br J Ophthalmol 86(2):238-242

5. Hamanaka T, Kasahara K, Takemura T (2011) Histopathology of the trabecular meshwork and Schlemm's canal in primary angle-closure glaucoma. Investig Ophthalmol Vis Sci 52(12):8849-8861. doi:10.1167/iovs.11-7591

6. Leung CK, Cheung CY, Li H, Dorairaj S, Yiu CK, Wong AL, Liebmann J, Ritch R, Weinreb R, Lam DS (2007) Dynamic analysis of dark-light changes of the anterior chamber angle with anterior segment OCT. Investig Ophthalmol Vis Sci 48(9):4116-4122. doi:10.1167/iovs.07-0010

7. International Glaucoma Review (2018) http://www.e-igr.com/MR/index.php?issue=43\&MRid=79. Accessed 17 Jan 2018

8. Sihota R, Kumar S, Sidhu T, Midha N, Sharma A, Yadav S, Gupta V, Dada T (2018) Is combined mechanism glaucoma a distinct entity? Graefe's archive for clinical and experimental ophthalmology = Albrecht von Graefes Archiv fur klinische und experimentelle Ophthalmologie 256 (10):1961-1969. doi:10.1007/s00417-018-4050-5

9. Tham Y-C, Li X, Wong TY, Quigley HA, Aung T, Cheng C-Y (2014) Global prevalence of glaucoma and projections of glaucoma burden through 2040: a systematic review and meta-analysis. Ophthalmology 121(11):20812090. doi:10.1016/j.ophtha.2014.05.013

10. Zhao J, Solano MM, Oldenburg CE, Liu T, Wang Y, Wang N, Lin SC (2019) Prevalence of Normal-Tension Glaucoma in the Chinese Population: A Systematic Review and Meta-Analysis. Am J Ophthalmol 199:101-110. doi:10.1016/j.ajo.2018.10.017

11. Cho HK, Kee C (2014) Population-based glaucoma prevalence studies in Asians. Survey of ophthalmology 59(4):434-447. doi:10.1016/j.survophthal.2013.09.003

12. Iwase A, Suzuki Y, Araie M, Yamamoto T, Abe H, Shirato S, Kuwayama Y, Mishima HK, Shimizu H, Tomita G, Inoue Y, Kitazawa Y (2004) The prevalence of primary open-angle glaucoma in Japanese: the Tajimi Study. Ophthalmology 111(9):1641-1648

13. Sommer A, Tielsch JM, Katz J, Quigley HA, Gottsch JD, Javitt JC, Martone JF, Royall RM, Witt KA, Ezrine S (1991) Racial differences in the cause-specific prevalence of blindness in east Baltimore. N Engl J Med 325(20):1412-1417 
14. Rotchford AP, Johnson GJ (2002) Glaucoma in Zulus: a population-based cross-sectional survey in a rural district in South Africa. Arch Ophthalmol 120(4):471-478

15. He M, Foster PJ, Johnson GJ, Khaw PT (2006) Angle-closure glaucoma in East Asian and European people. Different diseases? Eye (London England) 20(1):3-12. doi:10.1038/sj.eye.6701797

16. Wang L, Huang W, Huang S, Zhang J, Guo X, Friedman DS, Foster PJ, He M (2018) Ten-year incidence of primary angle closure in elderly Chinese: the Liwan Eye Study. Br J Ophthalmol. doi:10.1136/bjophthalmol2017-311808

17. Saw SM, Gazzard G, Friedman DS (2003) Interventions for angle-closure glaucoma: an evidence-based update. Ophthalmology 110(10):1869-1878. doi:10.1016/s0161-6420(03)00540-2 quiz 1878 - 1869, 1930.

18. Shingleton BJ, Chang MA, Bellows AR, Thomas JV (1990) Surgical goniosynechialysis for angle-closure glaucoma. Ophthalmology 97(5):551-556

19. Tanihara H, Nishiwaki K, Nagata M (1992) Surgical results and complications of goniosynechialysis. Graefe's archive for clinical and experimental ophthalmology = Albrecht von Graefes Archiv fur klinische und experimentelle Ophthalmologie 230 (4):309-313

20. Wand M (1992) Argon laser gonioplasty for synechial angle closure. Archives of ophthalmology (Chicago, III: 1960) $110(3): 363-367$

21. Comparison of glaucomatous progression between untreated patients with normal-tension glaucoma and patients with therapeutically reduced intraocular pressures. Collaborative Normal-Tension Glaucoma Study Group (1998). Am J Ophthalmol 126 (4):487-497

22. Yang $\mathrm{CH}$, Hung PT (1997) Intraocular lens position and anterior chamber angle changes after cataract extraction in eyes with primary angle-closure glaucoma. J Cataract Refract Surg 23(7):1109-1113

23. Tham CC, Lai JS, Lam DS (2001) Changes in AC angle width and depth after IOL implantation in eyes with glaucoma. Ophthalmology 108(3):428-429

24. Hayashi K, Hayashi H, Nakao F, Hayashi F (2000) Changes in anterior chamber angle width and depth after intraocular lens implantation in eyes with glaucoma. Ophthalmology 107(4):698-703

25. Greve EL (1988) Primary angle closure glaucoma: extracapsular cataract extraction or filtering procedure? International ophthalmology 12(3):157-162

26. Keenan TD, Salmon JF, Yeates D, Goldacre M (2009) Trends in rates of primary angle closure glaucoma and cataract surgery in England from 1968 to 2004. Journal of glaucoma 18(3):201-205.

doi:10.1097/IJG.0b013e318181540a

27. Wishart PK, Atkinson PL (1989) Extracapsular cataract extraction and posterior chamber lens implantation in patients with primary chronic angle-closure glaucoma: effect on intraocular pressure control. Eye 3(Pt 6):706712. doi:10.1038/eye.1989.109

28. Roberts TV, Francis IC, Lertusumitkul S, Kappagoda MB, Coroneo MT (2000) Primary phacoemulsification for uncontrolled angle-closure glaucoma. J Cataract Refract Surg 26(7):1012-1016

29. Hayashi K, Hayashi H, Nakao F, Hayashi F (2001) Effect of cataract surgery on intraocular pressure control in glaucoma patients. J Cataract Refract Surg 27(11):1779-1786

30. Jacobi PC, Dietlein TS, Luke C, Engels B, Krieglstein GK (2002) Primary phacoemulsification and intraocular lens implantation for acute angle-closure glaucoma. Ophthalmology 109(9):1597-1603

31. Khokhar S, Sindhu N, Pangtey MS (2002) Phacoemulsification in filtered chronic angle closure glaucoma eyes. Clin Exp Ophthalmol 30(4):256-260 
32. Ming Zhi Z, Lim AS, Yin Wong T (2003) A pilot study of lens extraction in the management of acute primary angle-closure glaucoma. Am J Ophthalmol 135(4):534-536

33. Kubota T, Toguri I, Onizuka N, Matsuura T (2003) Phacoemulsification and intraocular lens implantation for angle closure glaucoma after the relief of pupillary block. Ophthalmologica Journal international d'ophtalmologie International journal of ophthalmology Zeitschrift fur Augenheilkunde 217(5):325-328. doi:10.1159/000071346

34. Shams PN, Foster PJ (2012) Clinical outcomes after lens extraction for visually significant cataract in eyes with primary angle closure. Journal of glaucoma 21(8):545-550. doi:10.1097/IJG.0b013e31821db1db

35. World Glaucoma Association Consensus Statement: Intraocular Pressure. The Netherlands: Kluger (2007)

\section{Tables}

\section{Table 1 Statistical information of patients in the two groups}

PACG: primary angle-closure glaucoma; CMG: combined-mechanism glaucoma; IOP: intro-ocular pressure; ACD: anterior chamber depth; MD: mean deviation; VF: visual field; RNFL: retina nerve fiber layer; AL: axial length

\begin{tabular}{|c|c|c|c|c|c|c|c|c|}
\hline Items & $\begin{array}{l}\text { Age } \\
\text { 『year》 }\end{array}$ & $\begin{array}{l}\text { IOP before } \\
\text { surgery } \\
\text { \mmHg冈 }\end{array}$ & $\begin{array}{l}\mathrm{ACD} \\
(\mathrm{mm})\end{array}$ & $\begin{array}{l}\text { MD of } \\
\text { VF }\end{array}$ & $\begin{array}{l}\text { Average } \\
\text { RNFL } \\
\text { thickness } \\
(\mu \mathrm{m})\end{array}$ & $\mathrm{C} / \mathrm{D}$ ratio & $\mathrm{AL}(\mathrm{mm})$ & $\begin{array}{l}\text { Synechia } \\
\text { degree }\end{array}$ \\
\hline CMG & $\begin{array}{l}63.42 \\
\pm 5.90\end{array}$ & $20.75 \pm 5.06$ & $1.98 \pm 0.28$ & $\begin{array}{l}24.37 \pm \\
6.54\end{array}$ & $\begin{array}{l}48 \rrbracket \\
00 \pm 14.00\end{array}$ & $0.87 \pm 0.06$ & $22.62 \pm 0.76$ & $3.96 \pm 1.14$ \\
\hline PACG & $\begin{array}{l}59.42 \pm \\
7.47\end{array}$ & $21.25 \pm 4.47$ & $1.94 \pm 0.21$ & $\begin{array}{l}20.01 \pm \\
8.25\end{array}$ & $57.25 \pm 14.10$ & $0.88 \pm 1.11$ & $22.46 \pm 0.76$ & $9.38 \pm 1.99$ \\
\hline$P$ & 0.1586 & 0.8000 & 0.6989 & 0.4455 & 0.1211 & 0.3636 & 0.4689 & $<0.001$ \\
\hline
\end{tabular}

\section{Figures}



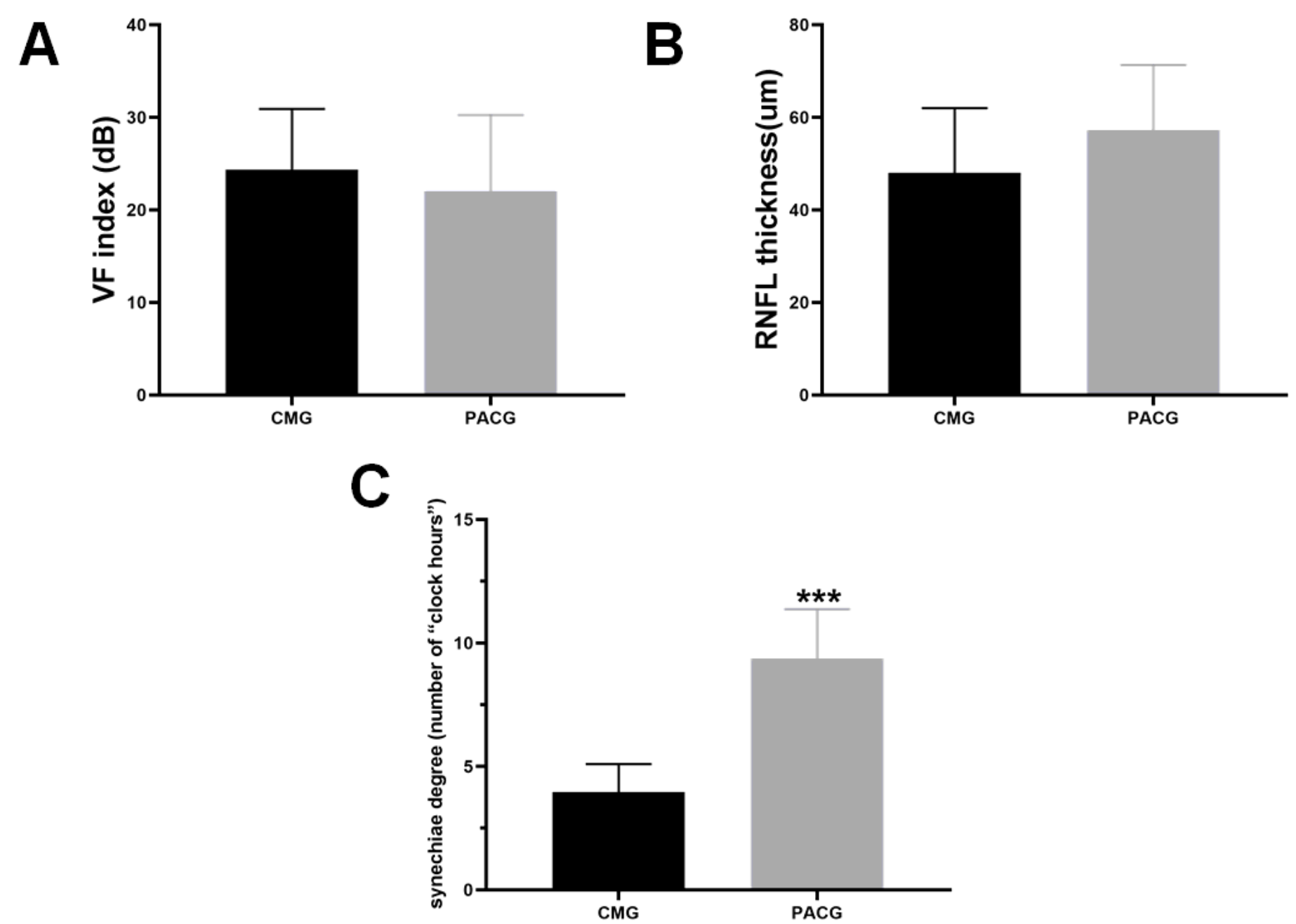

Figure 1

The extent of synechial angle closure in PACG group was more extended than that of CMG group. PACG: primary angle-closure glaucoma; CMG: combined-mechanism glaucoma; VF: visual field; RNFL: retina nerve fiber layer; $* \star \star: P$ $<0.001$

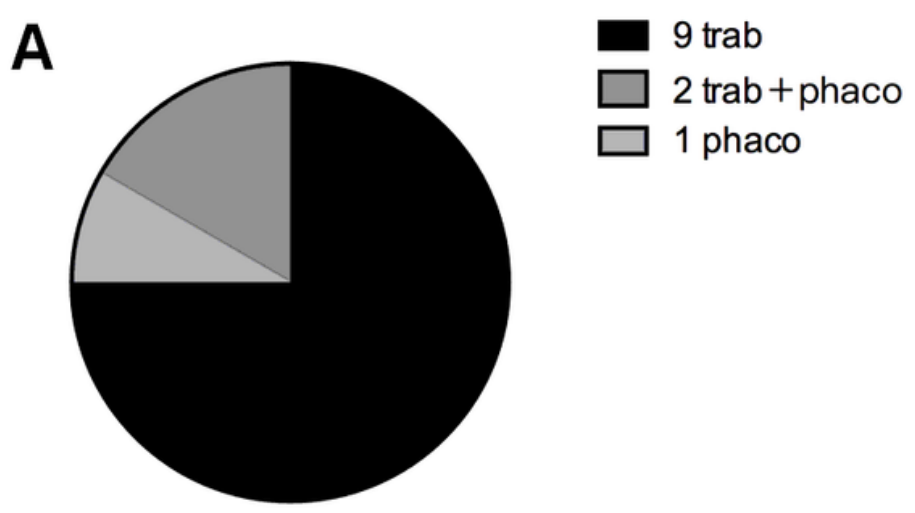

CMG group Total=12

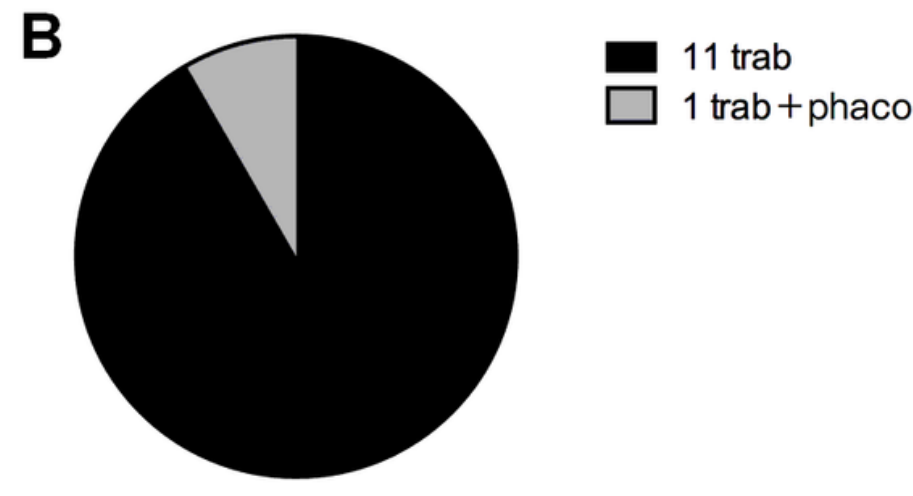

PACG group Total=12

Figure 2 
Surgical types of the two groups. PACG: primary angle-closure glaucoma; CMG: combined-mechanism glaucoma; trab: trabeculectomy

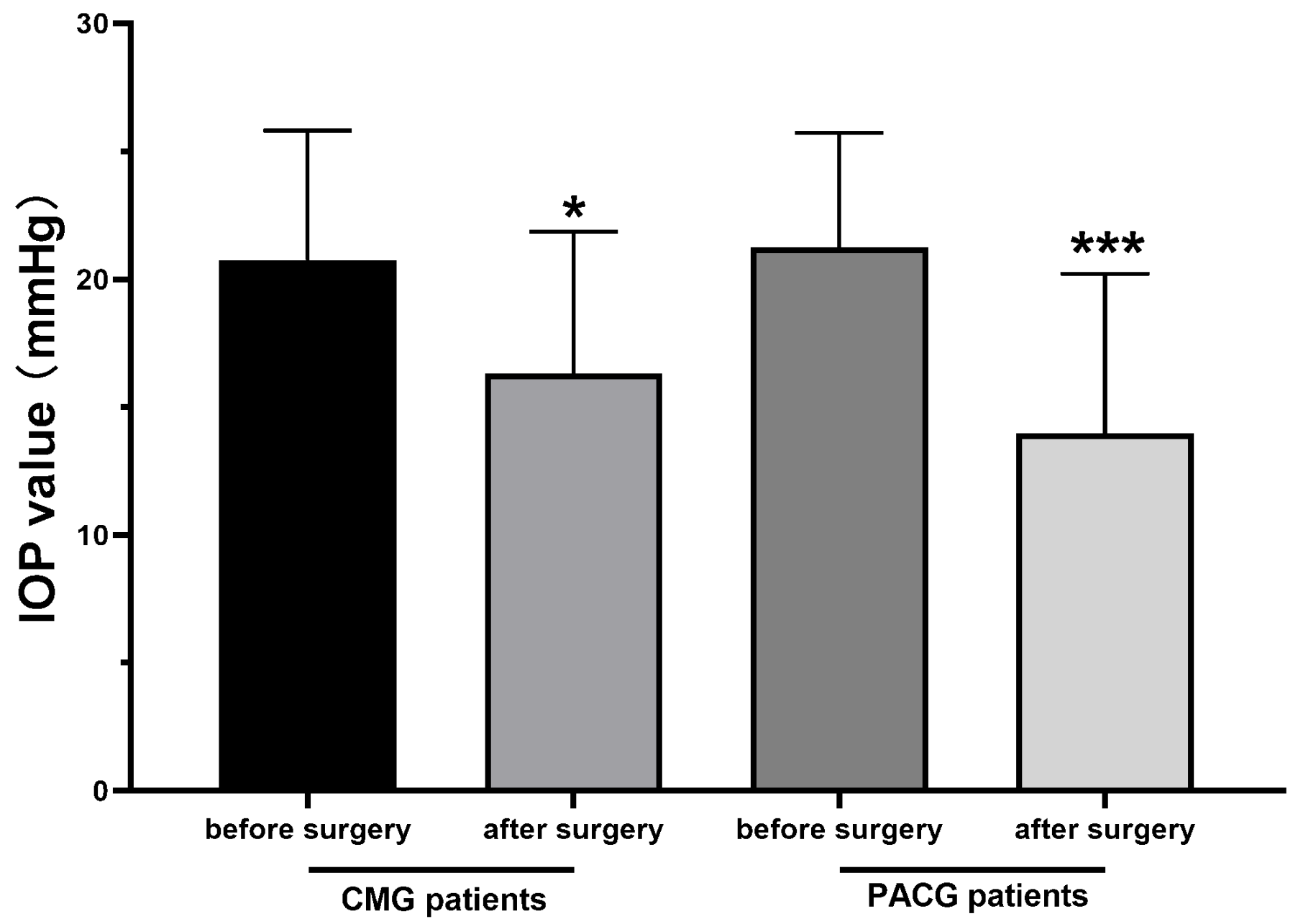

Figure 3

IOP decreased after surgery for both the two groups. PACG: primary angle-closure glaucoma; CMG: combinedmechanism glaucoma; IOP: intro-ocular pressure; *: $\mathrm{P}<0.05$; ***: $\mathrm{P}<0.001$ 